\title{
Analysis of the General Tool Life Function of Cutting Tools by Application of the Catastrophe-Theory
}

\author{
Zoltán Pálmai \\ University of Miskolc \\ János Kundrák ( $\sim$ kundrak@uni-miskolc.hu ) \\ University of Miskolc \\ Csaba Felhő \\ University of Miskolc
}

\section{Research Article}

Keywords: general tool life function, catastrophe-theory, turning, non-metallic deposition on tool

Posted Date: November 30th, 2021

DOI: https://doi.org/10.21203/rs.3.rs-1073955/v1

License: (1) This work is licensed under a Creative Commons Attribution 4.0 International License.

Read Full License 


\title{
Analysis of the general tool life function of cutting tools by application of the catastrophe-theory
}

\author{
Zoltán Pálmaiª, János Kundrák ${ }^{1 b, *}$, Csaba Felhő1c \\ (1) Institute of Manufacturing Science, University of Miskolc, 3515 Miskolc-Egyetemváros, Hungary \\ E-mail: apalmayz@t-online.hu, bkundrak@uni-miskolc.hu, cc csaba.felho@uni-miskolc.hu. Tel: a,b,c+3646565160
}

\section{Abstract}

Production technology planning requires information on tool life $\mathrm{T}$ and its relation to cutting speed v. As the Taylor formula often cannot be linearized on an lg-lg scale, a general tool life function has been developed for describing a $\mathrm{v}$-T function with a convex-concave part. Using catastrophe theory, an analogy is established between the general tool life function and the cusp catastrophe, allowing topological mapping of the general $\mathrm{v}$-T function. Results were verified by machinability tests in the turning of C35 and C60 conventional and specially deoxidized C-steels during steelmaking. It was found that in the convex-concave section of this function, 2-3 cutting speeds can be selected for a given tool life, which is advantageous for harmonizing tool changes in multi-operation technology.

Keywords: general tool life function, catastrophe-theory, turning, non-metallic deposition on tool

\section{Introduction}

More results are already known for the application of catastrophe theory in machining. This is due to the fact that several aspects of chip removal may be characterized by a sudden transformation of the gradual change into an abrupt/transient motion. On the one hand, as known, structural metals are characterized by the property of increasing their resistance to deformation during plastic deformation. On the other hand, the increase in temperature due to the thermal action of the deformation work reduces this resistance, i.e., it is a negative feedback from energetic point of view. A consequence may be thermal instability leading to the formation of so-called lamellar chips. Following Recht's work [1], this has been extensively studied by several researchers, including Komanduri and von Turkovich [2]. Catastrophe theory can also be used to study forming with high deformation. Klamecki [3] used this possibility in the shear zone by applying the principle of energy minimum, demonstrating that the phenomena he studied in chip formation can be described by the properties of Thom's cusp catastrophe [4]. The minimum energy surface is folded/back-bending and the system state changes along it. This can lead to sudden big changes. A similar finding was made by Miernik [5], who described the deformations in the chip root by a third- and fifth-degree polynomial using the principle of minimum energy. The former is a peak disaster with one degree of freedom, and the latter is a "butterfly catastrophe" with two degrees of freedom, with a double recoil on its energy surface.

Luo [6] reported an improvement in turning and end milling aimed at predicting tool wear through continuous monitoring of its condition. In evaluating the experimental results, the tools of catastrophe theory were utilized in such a way that the measurement data were processed with a cubic polynomial with variable parameters. The monitoring system thus designed made it possible to predict tool wear with more than $95 \%$ confidence.

Catastrophe theory has been successfully applied by several researchers in the case of non-free cutting. Here, the combined effect of the two shear zones prevails, and Shi et al. [7] investigated the direction of chip ejection using catastrophe theory. It was found that the chip ejection angle

\footnotetext{
${ }^{*}$ Corresponding author.
} 
differs if this event occurs as a result of decreasing or increasing the $\mathrm{a} / \mathrm{f}$ chip ratio. With a more detailed analysis, Cui et al. [8] and Zhu et al. [9] came to the same conclusion.

This report describes the work aimed at analyzing the general lifetime function of cutting tools using the tools of catastrophe theory. It has been proved that there is an analogy between the phenomena studied by the catastrophe theory and the technological dependence of the wear and tool life of the cutting tool, which can be successfully used for the qualitative analysis of cutting theory and practical manufacturing technology.

\section{About the general tool life function}

The Taylor formula is commonly used to calculate the tool life of cutting tools. This, as is known, is an empirical fractional hyperbola which, by default, describes the relationship between cutting speed $\mathrm{v}$ and tool life $\mathrm{T}$, preferably as a linear function on lg-lg scale diagrams (see the Ttrad in Fig. 1). But not always. Chip removal is essentially a very complex technological process, in strong interaction with the properties of the machined material and the tool. Of particular importance in this respect is the empirical fact that the load conditions of the tool and the abrasive effect on the surface change significantly under certain technological conditions.

The consequence of this is that in such cases the $\mathrm{v}$ - $\mathrm{T}$ relationship is described by the Taylor formula only in a limited interval, e.g. in Fig. 1 at the $v>40 \mathrm{~m} / \mathrm{min}$ section of the v-T curve. In the example, at a speed of $\mathrm{v}<40 \mathrm{~m} / \mathrm{min}$, the $\mathrm{v}$-T relation can be quite different, forming a convex and concave section from below (see Trec on Fig. 1).

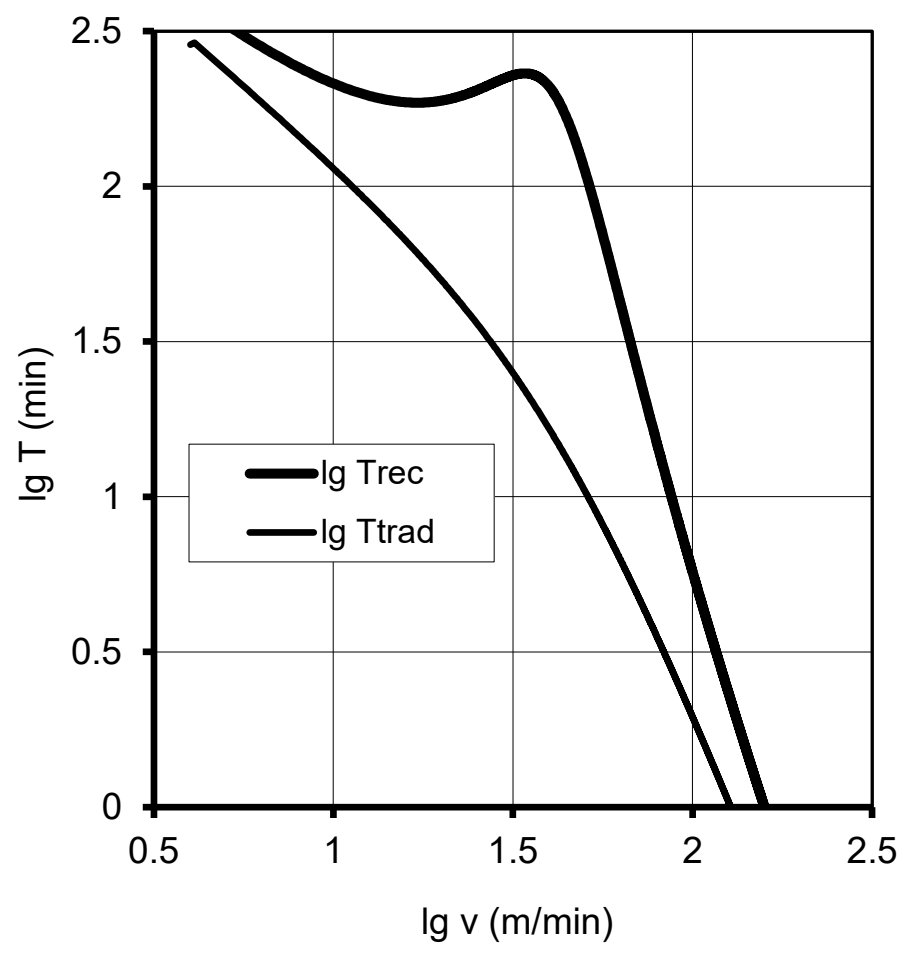

Fig. 1. Dependence of cutting tool life on cutting speed over a wide speed range (Ttrad: traditional; Trec: recommended) 
Several proposals have been made to solve this problem. The type of function proposed by Kundrák proved to be advantageous for theoretical analysis and practical application [10]:

$$
T=\frac{C_{1}}{v^{3}+C_{2} v^{2}+C_{3} v}
$$

An important feature of this function is that there is a well-utilized relationship between its two local extremes and the constant C2, C3 [10-13]:

$$
\begin{aligned}
& v_{\text {min,max }}=\frac{1}{3}\left(\mathrm{C}_{2} \pm \sqrt{\mathrm{C}_{2}{ }^{2}-3 \mathrm{C}_{3}}\right) \\
& \mathrm{C}_{2}=\frac{3}{2}\left(\mathrm{v}_{\min }-\mathrm{v}_{\max }\right) \\
& C_{3}=3 v_{\text {min }} v_{\text {max }}
\end{aligned}
$$

The section of the general tool life function, the nature of which is very different from the Taylor function, deserves special attention from a theoretical and technological point of view. This can be followed by the function (1) by the expedient choice of the constants $C_{i}(I=1,2,3)$. To illustrate this, Figure 2 shows the effect of $C_{2}$. Of course, the $C_{3}$ constant has a similar effect.

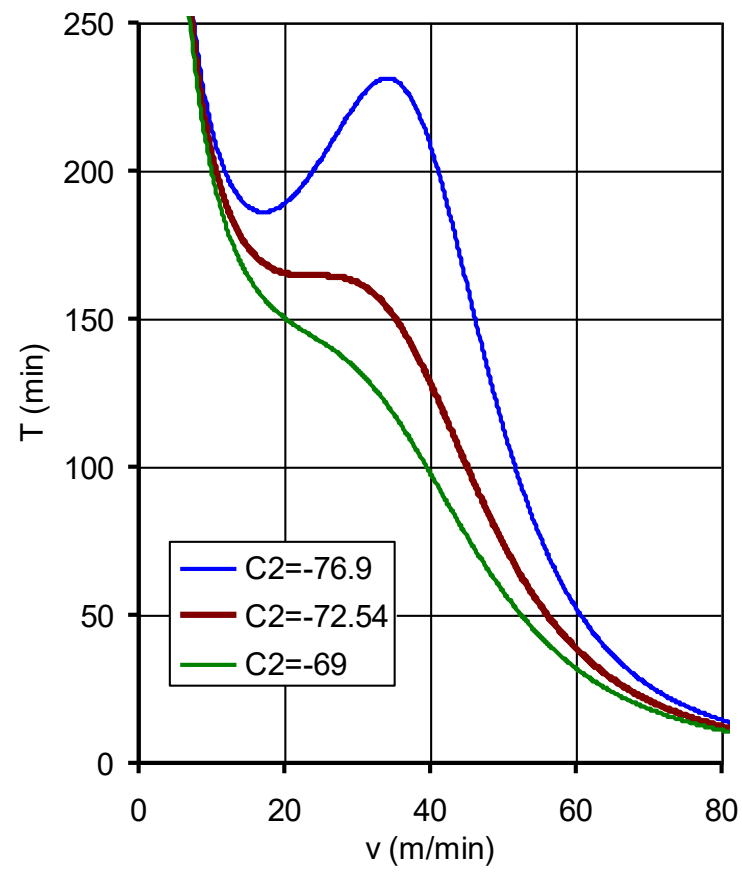

Fig. 2. Characteristics of the general tool life function (1) at three characteristic values of the constant $\mathrm{C}_{2}(\mathrm{C} 1=2330000, \mathrm{C} 3=1755)$ 
As an example, the general tool life curve for hard turning of the inner cylindrical surface of a hardened ball bearing ring of three diameters is shown in Fig. 3. [10].

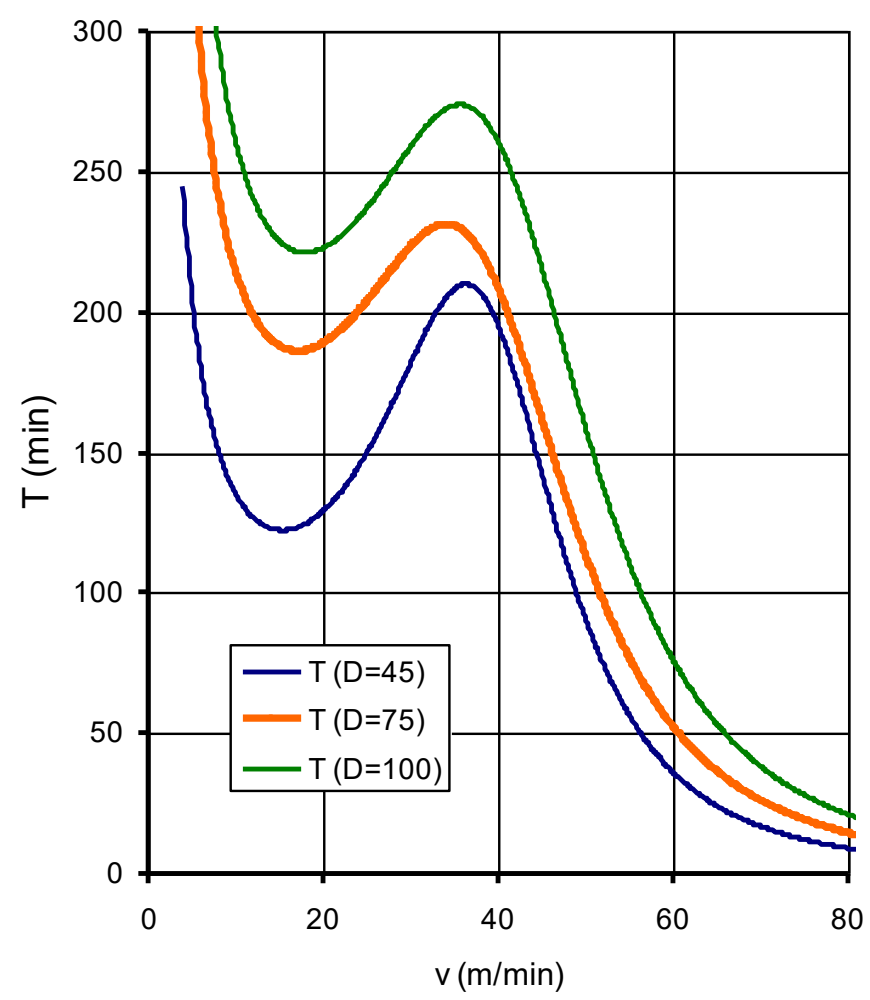

Fig. 3. General tool life function for hard turning of a hardened ball bearing ring [10-13] $\left(\mathrm{a}_{\mathrm{p}}=0.15 \mathrm{~mm}, \mathrm{f}_{\mathrm{z}}=0.05 \mathrm{~mm} / \mathrm{rev}\right.$, tool: Composite 10$)$

Table 1. The constants of the general tool life functions of the v-T curves in Figure 3

\begin{tabular}{llll}
$\mathrm{D} \mathrm{mm}$ & $\mathrm{C}_{1}$ & $\mathrm{C}_{2}$ & $\mathrm{C}_{3}$ \\
\hline 45 & 1380000 & -71.3 & 1494 \\
75 & 2330000 & -76.9 & 1755 \\
100 & 3180000 & -80.45 & 1923
\end{tabular}

An important feature of function (1) is that a third degree polynomial can be fitted to the middle section of Fig. 2, which illustrates the effect of the constant $\mathrm{C}_{2}$. This fit is shown in Fig. 4 for the velocity interval $\mathrm{v}=4-50 \mathrm{~m} / \mathrm{min}$ for the curve corresponding to the hole turning of diameter $\mathrm{D}=75 \mathrm{~mm}$ shown in Fig. 2 . 


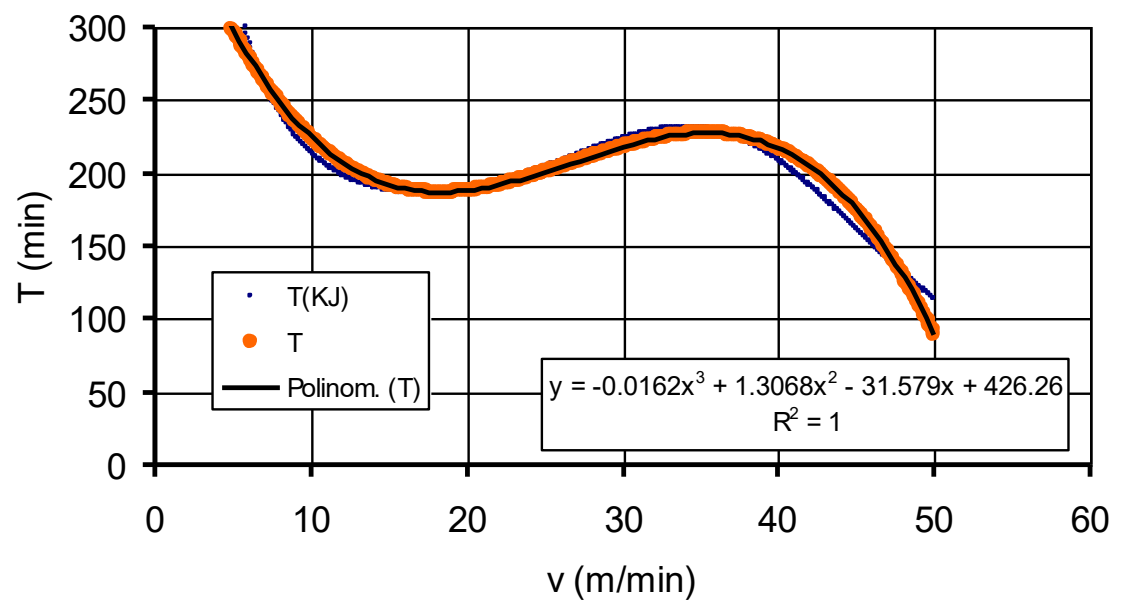

Fig. 4. Fitting of the general tool life function to a third-degree polynomial in the velocity range $\mathrm{v}=4-50 \mathrm{~m} / \mathrm{min}$ (see Fig. 2, $\mathrm{D}=75 \mathrm{~mm}$ )

The good fit can also be established by visual inspection, which in the present case is also shown by the Pearson number $\mathrm{R}^{2}=1$. A more detailed study also supports this finding, as shown in Figure 5.

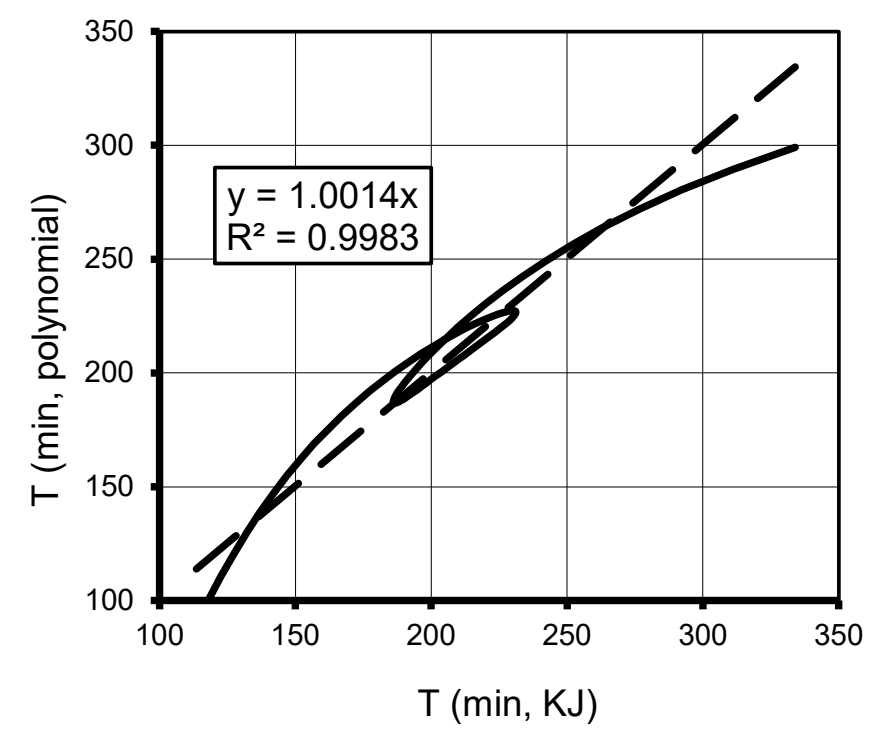

Fig. 5. Correlation of the general tool life function (1) and the third-degree polynomial for the case shown in Fig. 4.

Function (1) is also useful in the application scope of the Taylor formula. In the section of the v-T curve where the Taylor formula describes the velocity-tool life relationship well, Function (1) can also be applied with good agreement. Figure 6 shows this in this case at a constant $C_{2}=0$. Fig. 7 illustrates how the correlation between Function (1) and the linear lgv-lgT function can be increased by changing this constant $\mathrm{C}_{2}$. 


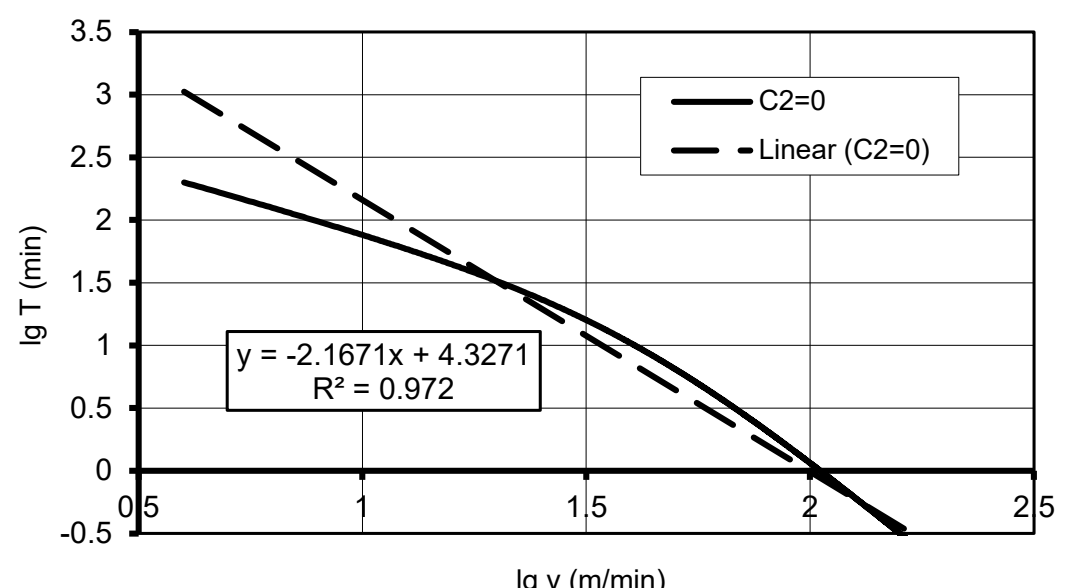

Fig. 6. Fitting of formula (1) to the Taylor function at a constant $C_{2}=0$

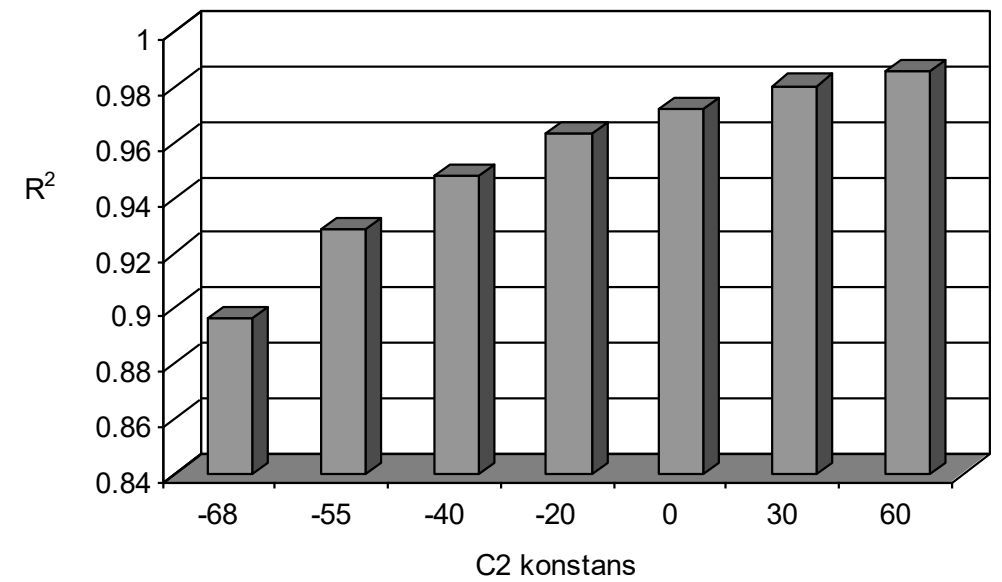

Fig. 7. The closeness of the relationship between the general tool life function (1) and the linear $\lg \mathrm{v}-\lg \mathrm{T}$ function as a function of the constant $\mathrm{C}_{2}$

In the section of the function curves $v$-T where the cutting speed $v_{\max }<\mathrm{v}$ (see e.g. Fig. 3), in principle the Taylor formula could also be applied. The two functions can be compared using the exponent -k of the Taylor formula. As it is known,

$$
v T^{-\frac{1}{k}}=C
$$

In the velocity range $\mathrm{v} \rightarrow \mathrm{v}+\Delta \mathrm{v}$, a change in tool life $\mathrm{T} \rightarrow \mathrm{T}+\Delta \mathrm{T}$ occurs. With these, the exponent $-\mathrm{k}$ can be calculated as a function of the speed $\mathrm{v}$ by the following formula:

$$
-k(v)=\frac{\lg \frac{T}{T+\Lambda T}}{\lg \frac{v}{v+\Lambda v}}
$$


The $\mathrm{k}(\mathrm{v})$ curves determined from the data in Table 1 are shown in Figure 8 . This curve apparently has a limit, $\mathrm{v}_{\text {lim }} \approx 3.2$.

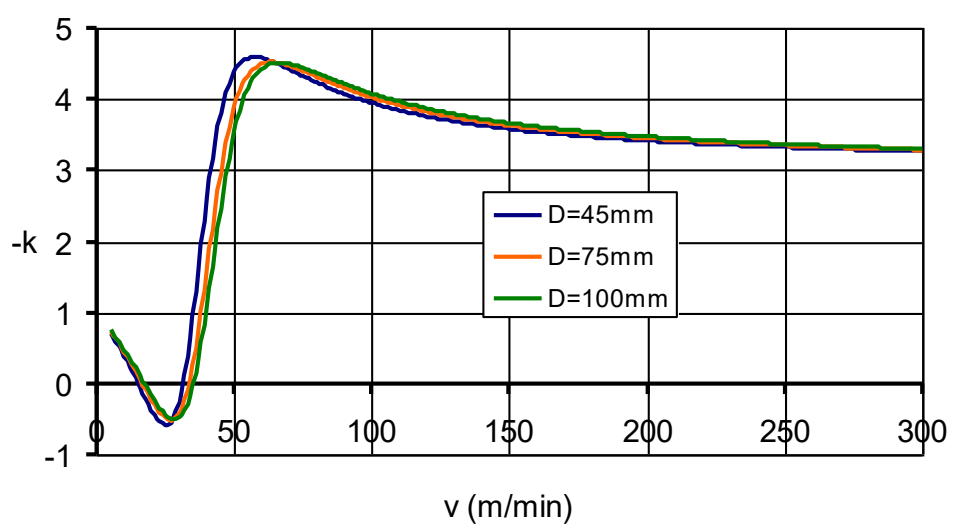

Fig. 8. The function $\mathrm{k}(\mathrm{v})$ showing the relationship between the general tool life function (1) and the Taylor formula (5) with the constants of Table 1.

These findings prove valid across a wide range of technological versions. Given that catastrophetheory analyses often use third-order polynomials, the tools of this theory offer useful help in analyzing the characteristics of the general tool life function.

\section{Catastrophe-theory analogy of the general tool life function}

In practice, it may be necessary to optimize time-consuming tool changes in sub-operations of a multi-stage technological task when harmonization of tool life is required. In such cases, the correct choice of cutting speed is the solution, i.e. the inverse of Fig. 2 can be used.

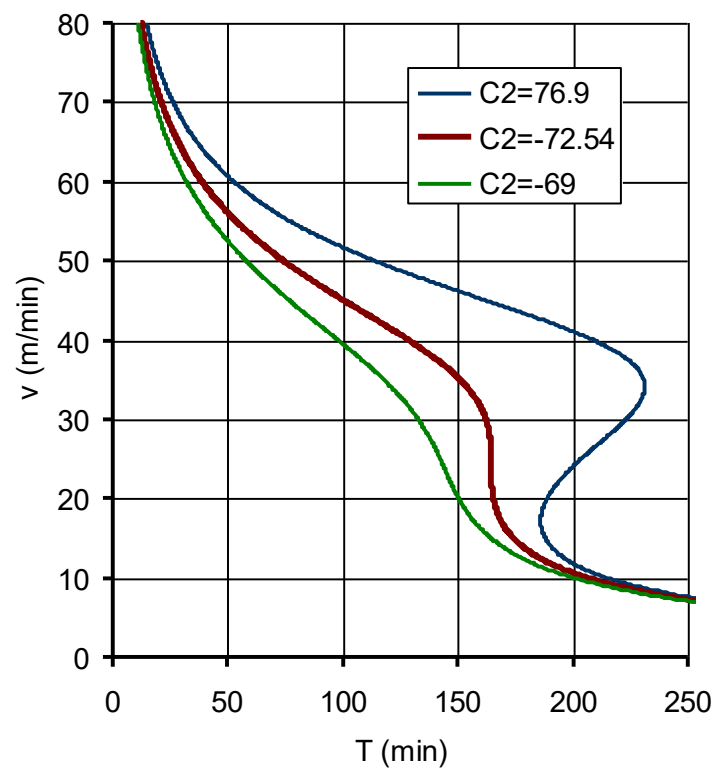

Fig. 9. Inverse of Fig. 2: Dependence of cutting speed $v$ on tool life $\mathrm{T}$ at three characteristic values of constant $\mathrm{C}_{2}$ 
The points $v_{i}=f\left(T_{i}\right)$ of the curves shown in Figure 9 can be calculated from the following equation by rearranging (1):

$$
v^{3}+C_{2} v^{2}+C_{3} v-\frac{C_{1}}{T}=0
$$

This equation can be written in the general form as:

$$
v^{3}+a v^{2}+b v+c=0
$$

Here $\mathrm{a}=\mathrm{C}_{2}, \mathrm{~b}=\mathrm{C}_{3}$ and

$$
c=-\frac{C_{1}}{T}
$$

$\mathrm{C}$ is thus a system parameter that is a function of an arbitrarily selected tool life $\mathrm{T}$ and determines the nature of the solution of Equation (8). According to the Cardano method used to solve cubic equations, by expedient transformation Equation (10) can be brought to the form [14]

$$
v^{3}+p v+q=0
$$

where:

$$
\begin{aligned}
& p=C_{3}-\frac{C_{2}{ }^{2}}{3} \\
& q=2\left(\frac{C_{2}}{3}\right)-\frac{C_{2} C_{3}}{3}+c
\end{aligned}
$$

Here $p$ and $q$ are system parameters. It can be seen from (10) that the parameter $q$ is the one with which the change of the cutting speed $\mathrm{v}$ as a function of the tool life $\mathrm{T}$ can be analyzed. The structure of the solutions of Equation (10) using the discriminant

$$
D=4 p^{3}+27 q^{2}
$$

of the quadratic equations is illustrated in Fig. 10: 


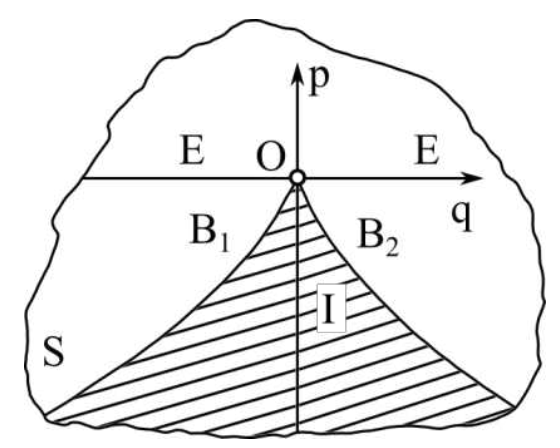

Fig. 10. Structure of the solutions of Equation (8)

Here, in the p-q coordinate system, the boundary curves $B_{1}$ and $B_{2}$ divide the field into two parts. By rearranging

$$
4 p^{3}+27 q^{2}=0
$$

which is expressed from (13),

$$
q_{\text {limit }}=\sqrt{-4\left(\frac{p}{3}\right)^{3}}
$$

is obtained, with which the boundary curves $B_{1}$ and $B_{2}$ can be calculated. In the case where the extreme values of the $\mathrm{v}$ - $\mathrm{T}$ curve slip together, i.e. $\mathrm{v}_{\min }=\mathrm{v}_{\max }$, then the discriminant of the quadratic equation in formula (2) is $D=0$. This results in:

$$
C_{3}=\frac{C_{2}{ }^{2}}{3}
$$

In the case of $v_{\min }=v_{\max }$, given the situation $\mathrm{p}=\mathrm{q}=0$, the function $\mathrm{T}-\mathrm{v}$ also has an inflection, which can be calculated from the second differential quotient of Function (1):

$$
v_{\text {origo }}=v_{\text {inf } l}=-\frac{C_{2}}{3}
$$

The consequence of $\mathrm{p}=\mathrm{q}=0$ is also that using (11) and (12):

$$
T_{\text {origo }}=T_{\text {inf } l}=-27 \frac{C_{1}}{C_{2}{ }^{3}}
$$

As a result, the cutting speed $v$ in the coordinate system $(p, q, v)$ is shown in Fig. 11. From the solution of the cubic equations, it is known that for $\mathrm{D}<0$, the equation has three real roots, which correspond to the dotted field $I$ in Fig. 10 and the points $A_{1}, A_{2}$ and $A_{3}$ in Fig. 11.

When $D>0$, there is a real and a conjugate complex root, and when $D=0, p \neq 0, q \neq 0$, then two real roots coincide. These are the bifurcation lines of the system, denoted by $B_{1}$ and $B_{2}$ in Figures 10 and 11 . Here the boundary curves shown in the p-q plane are the projections of the array 
contour of v-curves. If $\mathrm{D}=0, \mathrm{p}=\mathrm{q}=0$, then all three solutions coincide, and this is the origin of the $\mathrm{p}$ - $\mathrm{q}$ coordinate system. These characterize important features of the $\mathrm{v}$ - $\mathrm{T}$ tool life relationship of cutting tools. From the point of view of the roots of Equation (10), according to the geometric interpretation and representation, this shows how the point $(p, q)$ is located in the plane $p$-q. The nature of curve $\mathbf{s}_{1}$ in Figure 11 corresponds to the curve for constant $C_{2}=76.9$ and curve $\mathbf{s}_{2}$ for the constant $C_{2}=69$. The origin of Figures 10 and 11 is a special point, as shown in Figures 8 and 11 by the curve $\mathbf{s}$ for the constant $\mathrm{C}_{2}=-72.54$.

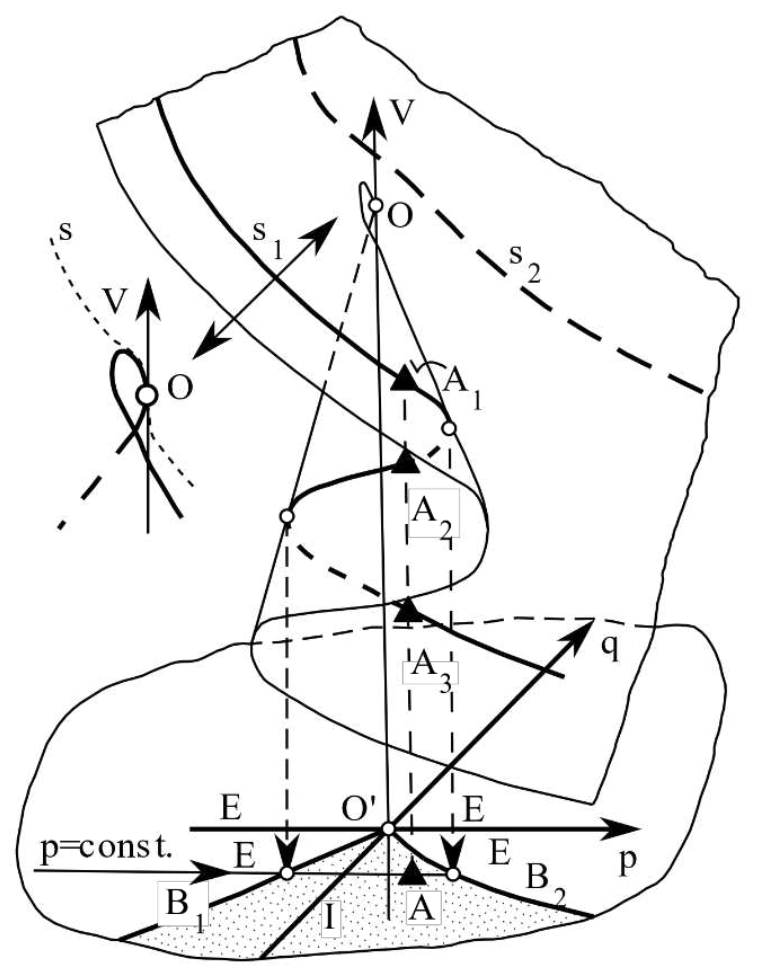

Fig. 11. The cutting speed $v$ as a function of the control parameters $p, q$

Figures 10 and 11 give a good overview of the mapping of $v-T \rightarrow p$-q by the control parameters $p$ and q. The mapping of the v-T functions shown in Fig. 3 according to (11), (12) is illustrated in Fig. 12 in the p-q coordinate system.

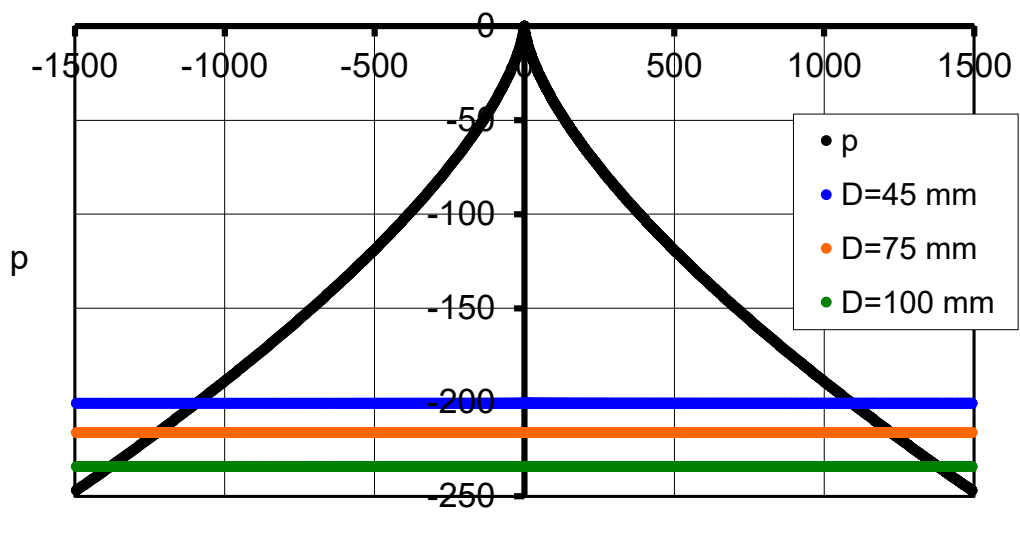

q

Fig. 12. Projections of the v-T curves in Figure 3 in the p-q plane 


\section{Catastrophe-theory analogy of the impact of material accumulation on the cutting tool}

Sections 2 and 3 presented the analogy between tool life - which is important for both theory and practice - and technological parameters, primarily cutting speed $\mathrm{v}$. While it is true that catastrophe theory deals essentially with the dynamic behavior of structures, it is sufficient to think only of Zeeman machines [14], which use concepts derived from a potential function. Such, of course, cannot be interpreted when examining the tool life. However, a strong analogy can be established, which makes the use of the tools of catastrophe theory usable in this field of technology as well.

This analogy is confirmed by studies aimed at determining the effect of occasional non-metallic or metallic material accumulation on the cutting tool.

Particularly important in this respect are the material processes that take place on the tool surface within certain limits of the technological parameters, in particular the cutting speed v.

Two essentially similar phenomena are particularly worth highlighting. These are deposits of material on the tool that can destroy but also protect the surface of the tool. As a result, either harmful metallic (built-up edge) or useful non-metallic material deposition occurs on the tool surface. The type of function (1) shown in Figure 1 characterizes the tool life well.

A phenomenon is known where a non-metallic material builds up on the tool. [15]. This is created in the material of the workpiece from deoxidation products remaining during steelmaking [16]. The non-metallic layer can dramatically slow down tool wear. Figure 13a shows a picture of a conventional crater wear. Figure 13b shows, a tool face after turning with the same time and the same technology, on which this special non-metallic layer has formed. This non-metallic deposition also occurs on the flank of the tool (Figure 14). It can be seen that this phenomenon can greatly increase the tool life.

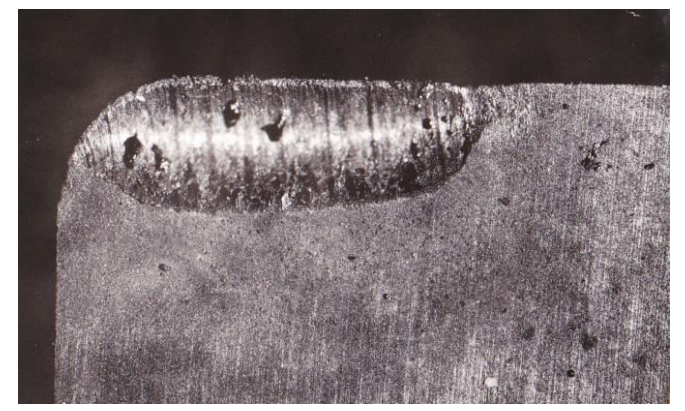

a)

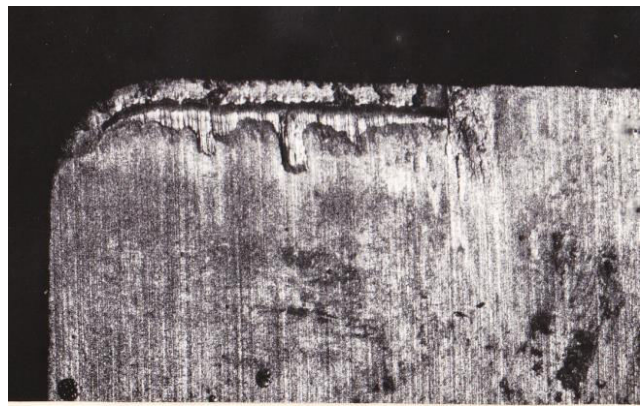

b)

Fig. 13. Effect of contact between cutting tool and chip: (a) normal crater wear, (b) occasionally a non-metallic protective layer

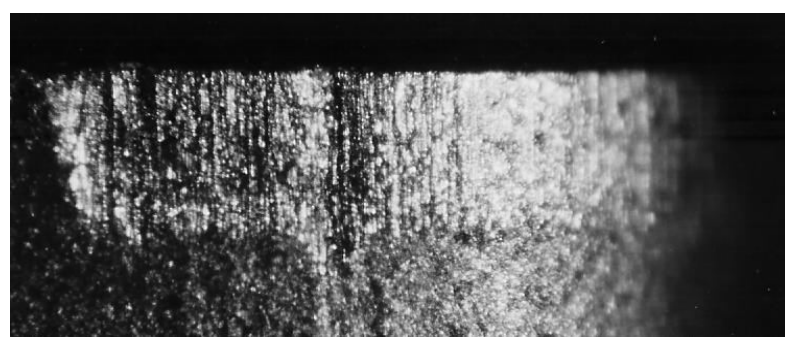

a)

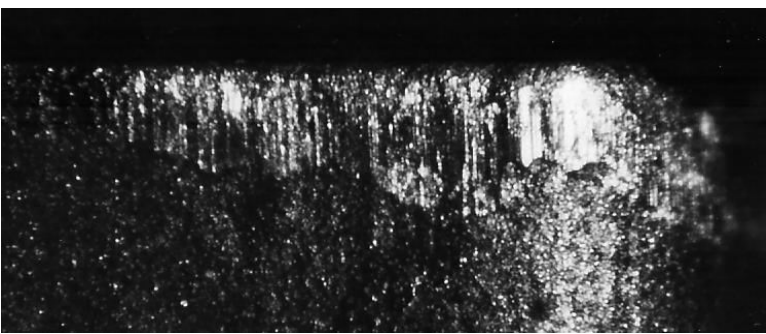

b)

Fig. 14. Occasional non-metallic deposits on the flank of the cutting tool: (a) normal flank wear,

(b) wear-slowing non-metallic material deposition 
The effect of non-metallic inclusions formed in the steel material of the workpiece (this is also possible for other metals) was investigated for several versions of non-alloy C-steel marked C35 and C60. Important information for the evaluation of the results is that the steel was produced under large-scale conditions using oxygen-Siemens-Martin technology. It is known that the manufacturing process fundamentally determines the chemical composition of deoxidation products and inclusions remaining in the steel, and thus the strength and plasticity properties. Production batches weighed an average of 105 metric tons.

A cutting test of steel from three production batches of C60 grade batches is presented here. There were two experimental batches in which the standard deoxidation technology was changed. One had an Al overdose. The aim was to make the steel the cleanest available under operating conditions, with few inclusions remaining in the steel. In addition, the deoxidation products remaining in smaller quantities should be high Al content inclusions with high thermal strength. Turning of this steel certainly does not result in non-metallic deposits.

In the other experimental batch, a complex deoxidizing alloy was used, which caused the residual inclusions to be complex CaMgAl silicates, which are plastic under the conditions of warm forming. This steel is marked C60 HM (high machinability).

In addition to these two versions, one of the steel batches manufactured and tested under several normal operating conditions was selected whose machinability is among the results obtained for the two test materials. It is marked $\mathrm{C} 60 \mathrm{~N}$ (in this case this does not indicate normalization of the steel, the machined steel samples were in a rolled state). Chemical compositions of the investigated materials are given in Table 2.

Table 2. Chemical composition of C60 grade experimental steel batches

\begin{tabular}{lllllllll} 
Grade & Batch No. & $\mathrm{C}$ & $\mathrm{Mn}$ & $\mathrm{Si}$ & $\mathrm{S}$ & $\mathrm{P}$ & $\mathrm{Al}$ & $\mathrm{Cu}$ \\
\hline C60 & 47411 & 0.61 & 0.64 & 0.21 & 0.0 .24 & 0.023 & $0.192^{* *}$ & 0.23 \\
C60 HM* & 48469 & 0.62 & 0.77 & 0.23 & 0.025 & 0.018 & $0.01^{* * *}$ & 0.21 \\
C60 N & 77332 & 0.57 & 0.72 & 0.22 & 0.046 & 0.027 &. & 0.19
\end{tabular}

${ }^{*} \mathrm{HN}$ : High Machinability, ${ }^{* *}$ Over-deoxidized with $\mathrm{Al},{ }^{* * *}$ Deoxidized with complex SiCaMgAl alloy

Table 3. The constants of Equation (5) for turning the steels given in Table 2

\begin{tabular}{llll} 
Steel & $\mathrm{C}_{1}$ & $\mathrm{C}_{2}$ & $\mathrm{C}_{3}$ \\
\hline C60 & 9500000 & -50 & 2178 \\
C60 HM & 76900000 & -240.75 & 17923 \\
C60 N & 76900000 & -228 & 17923
\end{tabular}

The results of the tool life tests are summarized in Figure 15. The standard representation of v-T functions is usually on an lg-lg scale, so in addition to the linear-scale in Fig 15a, the figure also shows the standard lg-lg version (Figure 15b). The result of the $\mathrm{v}-\mathrm{T} \rightarrow \mathrm{p}-\mathrm{q}$ transformation is shown in Fig. 16. 


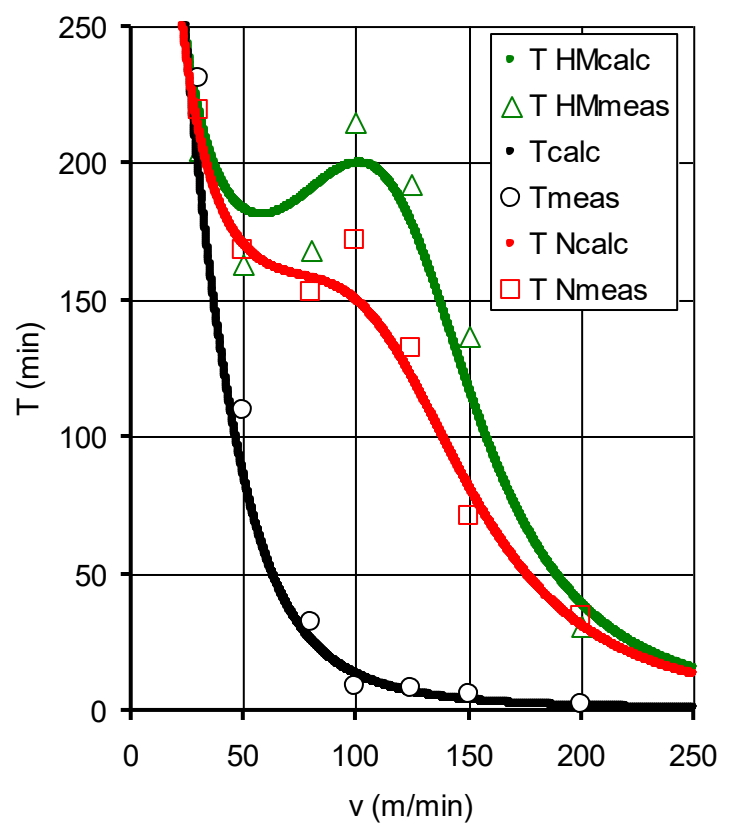

a)

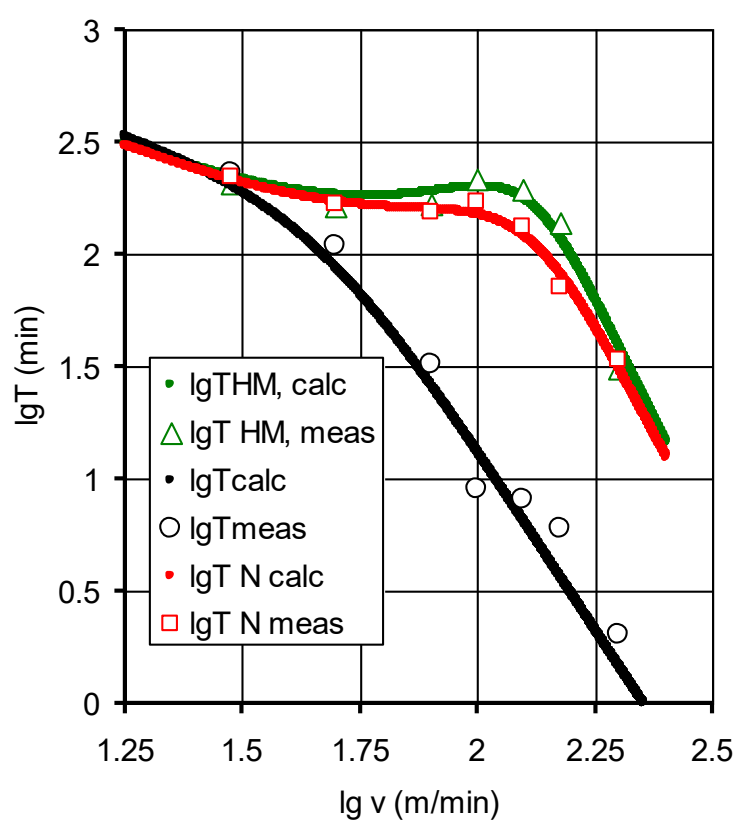

b)

Fig. 15. Tool life as a function of cutting speed when turning C60, C60 HM and C60N steel: (a) linear and (b) logarithmic coordinate system

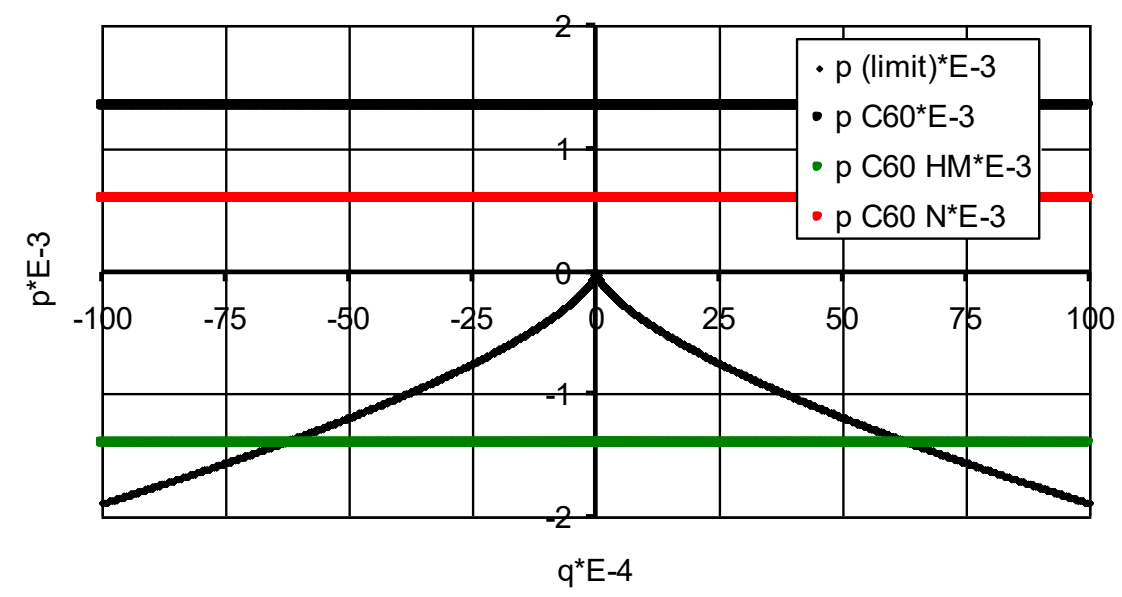

Fig. 16. Catastrophe theory summary of the results of the tool life tests performed on three C60 grade steels

The machinability of the steels was tested for 21 batches of regular operationally produced C35 grade C steel. The unalloyed C-steel was of C35 grade. Test specimens with a diameter of $\mathrm{D}=76 \mathrm{~mm}$ and a length of $700 \mathrm{~mm}$ were produced from rolled samples with a cross-section of $80 \times 80 \mathrm{~mm}^{2}$ taken from the production process. Machinability was tested by turning. Technological data were as follows: $\mathrm{v}=100,150$ and $200 \mathrm{~m} / \mathrm{min}, \mathrm{t}=5 \mathrm{~min}$, tool: P20, $\mathrm{f}=0.3 \mathrm{~mm} / \mathrm{rev}_{\text {., }} \mathrm{a}=2 \mathrm{~mm}, \gamma=-6^{\circ}, \gamma_{\mathrm{r}}=60^{\circ}$. Machinability was assessed by measuring VB average flank wear. The results were summarized in five groups and are shown in Table 4. 
Table 4. Results of the machinability test on samples taken from factory production at speeds $\mathrm{v}=100,150$ and $200 \mathrm{~m} / \mathrm{min}$

\begin{tabular}{lll} 
Group number & Flank wear classification & Number of cases \\
\hline I. & $\mathrm{VB}_{\mathrm{v}=100}<\mathrm{VB}_{\mathrm{v}=150}<\mathrm{VB}_{\mathrm{v}=200}$ & 7 \\
II. & $\mathrm{VB}_{\mathrm{v}=100}>\mathrm{VB}_{\mathrm{v}=150}$ & 4 \\
III. & $\mathrm{VB}_{\mathrm{v}=150}>\mathrm{VB}_{\mathrm{v}=200}$ & 2 \\
IV. & $\mathrm{VB}_{\mathrm{v}=150} \approx \mathrm{VB}_{\mathrm{v}=200}$ & 1 \\
V. & Uninterpretable or non-metallic protective deposit & 7
\end{tabular}

Group I include samples in which the flank wear increased, the tool life decreased monotonically as a function of cutting speed. In these cases, the Taylor formula can be used in the usual way. In Groups II and III there are essentially similar cases. They are characterized by the fact that the v$\mathrm{T}$ function does not monotonically decrease, so the general tool life function is required. The $\mathrm{v}-\mathrm{T}$ function includes both convex and concave curve segments, so the tool life $\mathrm{T}$ has a range at which a selected $\mathrm{T}_{\mathrm{i}}$ value has two or three cutting speeds.

A special case is classified in Group IV, in which the measured wear is visibly unchanged in the speed range $v=150-200 \mathrm{~m} / \mathrm{min}$. This suggests that for this steel batch, the machinability may be a special limit case; in Figures 10 and 11 the curve q-v passes through the origin (see curve s). For materials in Group I, $p>0$, while for Groups II and III $p<0$.

\section{Discussion}

One of the main advantages of catastrophe theory models is that they are able to predict the expected characteristics of the system over a wide range of interpretations, situations in which the cost and time required for total experimental measurement would be unfeasible in many cases. In the present case, the dynamics of the model does not appear directly in the relationship between the cutting speed $\mathrm{v}$ and the tool life $\mathrm{T}$, but in the change of the system parameters $\mathrm{p}$ and q. Catastrophe theory is usually used to analyze the motion of dynamic systems. Here, however, the application possibilities of a cutting technology model are presented through a demonstrable mathematical and topological analogy.

In dynamic systems, the principle of energy minimum, as shown in Fig. 11, can be represented as a back-folded surface in a topological approach. Quantities that describe the current state of a process can be considered as coordinates of a multidimensional space. The states of the studied system are represented in space by a hypersurface. The topological interpretation of the investigated system is a tool for the qualitative interpretation of the measurement results.

In the present case, of course, the principle of minimum energy does not apply. Yet the possibility of using the analogy has the potential to fit well the third-degree polynomial representing the cusp catastrophe to the convex-concave section of the general tool life function. The analogy therefore does not appear in that a gradual change suddenly becomes great. The drastic topographic change does not reflect a sudden change in state. In the present case, in relation to the cutting speed and the tool life - under definable conditions - a single current cutting speed value can have two or three tool life values. This is a natural consequence of the occasionally appearing connected convex and concave sections on the $\mathrm{v}$ - $\mathrm{T}$ curve. Thus, it may be the case that, under definable conditions, a technology can be selected in which a tool life can be set at two or three cutting speeds. In Figure 17, the area in which the function T(v) is multivalent is delimited by points A, B, C and D. For dynamic systems, the curve section B-C shown in the 
figure is considered to be an unstable position in which the system cannot be permanently. Structural stability is the guiding principle of catastrophe theory, introduced by Thom [4]. This means that the behavior of the system is not affected by small perturbations and the system remains stable. This, of course, cannot be interpreted here. The analogy is therefore not valid in this respect because in the model discussed in this study, all three stages, i.e., A-B, B-C, and C-D, are equivalent, and also stable in this sense. The model does not deal with sudden changes, but with the technological possibility that within definable limits, in this case in the field marked with points A-D in Fig. 17, it is possible to consider three technological versions. Thus, the tool life here is not the result of an investigation, but a parameter that helps to optimize the technological process. As a result, the application of catastrophe theory helps to optimize cutting technology by qualitatively describing the relationship between $\mathrm{v}$-T cutting speed and tool life, which is sometimes complicated.

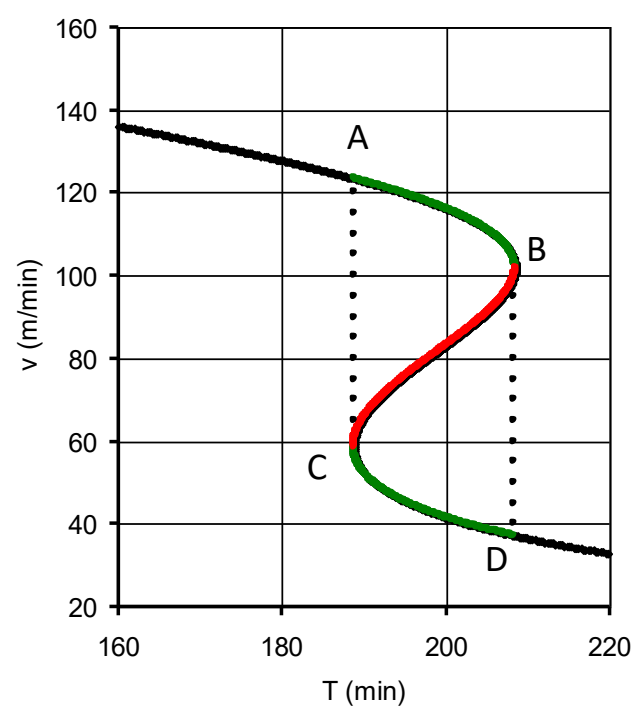

Fig. 17. A characteristic convex-concave section of the general T-v function

Special attention is paid to the environment around the origins of catastrophe theory (Fig. 11). This is also interesting in this analogical model. As can be seen in Fig. 11, at the working point v$\mathrm{T} \rightarrow \mathrm{q}-\mathrm{v}$ close to the origin, practically any cutting speed can be selected for the tool life $\mathrm{T}$ within an interval.

In the present case, the analogy of the cusp catastrophe was applied. There was no need to use higher order models. However, this would be possible, which would allow us to examine the interaction of several variables. For example, one of the cutting parameters cutting speed $v$, feed rate $f$, and tool life $T$ can be arbitrarily selected as the main variable of the model, and the other two as system parameters control its behavior. Such an analogy would require the use of a fifthorder polynomial instead of a third-order one, and its topography would lead to a doublecurved, two-pointed, so-called nightingale model.

\section{Summary}

The aim of this study was to summarize studies aimed at the functional analysis of the tool life of cutting tools. The widely known and used Taylor formula is unsuitable for describing cutting speed-tool life relationships that cannot be linearized on the lg-lg scale. Their graph also has a convex-concave section, for the description of which a commonly used tool life function has been 
developed. The applicability of the function has been demonstrated by turning hardened ball bearing rings [10].

It was found that the convex-concave section of the function can be well approximated by a cubic polynomial analogous to the mathematical description of the cusp catastrophe known from catastrophe theory. This made it possible to topologically map the $\mathrm{v}$-T tool life function and, on this basis, to analyze the properties of the function.

The analogy method was verified by turning C35 and C60 grade C steels. Two experimental steel batches weighing 105 tons were produced to study the v-T function relationship. One batch was over-deoxidized with Al to minimize the concentration of residual deoxidation products in the steel. These inclusions are brittle. The other experimental batch was treated with an alloy from which complex CaMgAl-silicate inclusions remained in the steel. These complex inclusions smear on the wear surfaces of the tool during cutting and reduce tool degradation. In this way, the Taylor curve obtained during the cutting of Al-deoxidized steel was distorted in the direction of the longer tool life, and a convex-concave section was formed on the v-T curve. The tests also included the turning of a batch of steel taken from factory production. The general tool life function fitted well to the $\mathrm{v}$-T curve obtained from all three measurement series. The catastrophe theory analogy allowed for a detailed analysis of these. The results were also confirmed by factory routine machinability tests performed on C35 grade steel. Statistical processing of the turning of the 21 batches also confirmed the applicability of the catastrophetheoretical analogy. It has been shown that in the convex-concave section of the general v-T curve, two or three cutting speeds can be selected for a currently desired tool life. This is advantageous for harmonizing tool change times in multi-operational processes.

\section{Declarations}

\section{Funding}

Not applicable

\section{Conflicts of interest/Competing interests}

Not applicable

\section{Availability of data and material}

All the data and materials are available upon request directly from the correspondent author.

\section{Code availability}

Not applicable

\section{Authors' contributions}

Zoltán Pálmai: main idea, theoretical foundation, literature review, data analysis, conclusions.

János Kundrák: theoretical considerations, tool life formula construction, data analysis, professional support, work management.

Csaba Felhő: overall text editing, text translation, making of the illustrations, figures, graphs, paper finalization.

\section{References}

[1] R.F. Recht, Catastrophic Thermoplastic Shear, ASME Journal of Applied Mechanics 31 (1964) 189193.

[2] R. Komanduri, B.F. von Turkovich, New observations on the mechanism of chip formation when machining titanium alloys. Wear 69 (1981) 179-188. 
[3] B.E. Klamecki, Catastrophe Theory Models of Chip Formation, Trans. of the ASME Journal of Engineering for Industry 104 (1982) 369-374.

[4] R. Thom, Structural Stability and Morphogenesis, W.A. Benjamin Inc., 1975

[5] M. Miernik, Application of the Catastrophe Theory to the Study of the Chip Formation Process, Mathematics 37(1) (1992) 3-7.

[6] Z. Luo, Study on catastrophe theory-based modelling and prediction of tool life, Chinese Journal of Mechanical Engineering 30(3) 1994 105-112.

[7] H. Shi, X. Weng, T. Tu, Bifurcation and Catastrophe in Metal-cutting Processes. Trans. of the ASME, Journal of Manufacturing Science and Engineering 120 (1998) 817-820.

[8] H. Cui, X. Wan, L. Xiong, Modelling of the catastrophe of cutting flow angle in the turning with double-edged tool with arbitrary rake angle based on catastrophe theory, The International Journal of Advanced Manufacturing Technology 104 (2019) 2705-2714. DOI: 10.1007/s00170-019-041141

[9] B. Zhu, Y.M.H. Xiao, X. Wan, L. Xio, Theoretical modelling and experimental verification of chip flow angle catastrophe in double-edged cutting considering non-linear effects, International Journal of Mechanical Sciences 172 (2020) 105394. DOI: 10.1016/j.ijmecsci.2019.105394

[10] J. Kundrak, The Scientific Principles of Increasing the Effectiveness of Inner Surfaces Cutting with CBN Tools, DSc dissertation, Kharkov, 1996

[11] A.G. Mamalis, J. Kundrák, M. Horváth, Wear and Tool Life of CBN cutting tools. International Journal of Advanced Manufacturing Technology 20 (2002) 475-479. DOI: 10.1007/s001700200180

[12] A.G. Mamalis, J. Kundrák, M. Horváth, On a novel tool life relation for precision cutting tools, Journal of Manufacturing Science and Engineering 127(2) (2005) 328-332. DOI: 10.1115/1.1794158

[13] J. Kundrak, Z. Pálmai, G. Varga, Analysis of Tool Life Functions is Hard Turning, Technical Gazette 27(1) (2020) 166-173. DOI: 10.17559/TV-20190712153727

[14] T. Poston, I. Stewart, Catastrophe Theory and its Applications, Pitman Publishing, London, 1978.

[15] Z. Pálmai, The effect of deoxidation of steel on machinability, Wear 38 (1976) 1-16. DOI: 10.1016/0043-1648(76)90195-2

[16] Z. Pálmai, The effect of a non-metallic material deposit in decreasing the wear of TiN-coated high speed steel cutting tools, Wear 95 (1984) 1-7. DOI: 10.1016/0043-1648(84)90185-6 